\title{
Generalized Solutions for Nonlocal Elliptic Equations and Systems with Nonlinear Singularities
}

\author{
Youtao Wang and Guangcun Lu \\ Laboratory of Mathematics and Complex Systems, School of Mathematical Sciences, Beijing Normal University, Ministry of Education, \\ Beijing 100875, China
}

Correspondence should be addressed to Guangcun Lu; gclu@bnu.edu.cn

Received 14 April 2015; Revised 4 June 2015; Accepted 7 June 2015

Academic Editor: Julio D. Rossi

Copyright (C) 2015 Y. Wang and G. Lu. This is an open access article distributed under the Creative Commons Attribution License, which permits unrestricted use, distribution, and reproduction in any medium, provided the original work is properly cited.

We use the topological degree method to study the existence of solutions for nonlocal elliptic equations (systems) with a strong singular nonlinearity.

\section{Introduction and Main Results}

Given $s \in(0,1)$, an integer $n>2 s$, and a bounded open set $\Omega$ of $\mathbb{R}^{n}$ with Lipschitz boundary, let $K: \mathbb{R}^{n} \backslash\{0\} \rightarrow(0,+\infty)$ be a function satisfying the following properties:

(i) $\gamma K \in L^{1}\left(\mathbb{R}^{n}\right)$ with $\gamma(x)=\min \left\{|x|^{2}, 1\right\}$.

(ii) There exists $\theta>0$ such that $K(x) \geq \theta|x|^{-(n+2 s)}$ for any $x \in \mathbb{R}^{n} \backslash\{0\}$.

(iii) $K(x)=K(-x)$ for any $x \in \mathbb{R}^{n} \backslash\{0\}$.

The so-called nonlocal elliptic operator $\mathfrak{S}_{K}$ is defined by

$$
\begin{array}{r}
\mathfrak{Q}_{K} u(x) \\
=\frac{1}{2} \int_{\mathbb{R}^{n}}(u(x+y)+u(x-y)-2 u(x)) K(y) d y, \\
x \in \mathbb{R}^{n} .
\end{array}
$$

In particular, when $K(x)=|x|^{-(n+2 s)}, \mathfrak{Q}_{K}$ is equal to the fractional Laplace operator $-(-\Delta)^{s}$ (up to normalization factors).

For a Carathéodory function $f: \Omega \times \mathbb{R} \rightarrow \mathbb{R}$, the following problem

$$
\begin{aligned}
\mathfrak{\Omega}_{K} \mathfrak{u}+f(x, u)=0 & \text { in } \Omega, \\
u=0 & \text { in } \mathbb{R}^{n} \backslash \Omega
\end{aligned}
$$

and its special case

$$
\begin{aligned}
(-\Delta)^{s} u & =f(x, u) \quad \text { in } \Omega, \\
u & =0 \quad \text { in } \mathbb{R}^{n} \backslash \Omega
\end{aligned}
$$

have been widely studied under various contexts; see a recent survey [1] for details.

1.1. Previous Work. Motivated by the work of Caffarelli and Silvestre [2], several authors have considered an equivalent problem of (3) by means of an auxiliary variable; see [2-6]. Precisely, let $(x, y)$ denote the points in $\mathscr{C}_{\Omega}:=\Omega \times(0, \infty) \subset$ $\mathbb{R}_{+}^{n+1}$ and $\partial_{L} \mathscr{C}_{\Omega}=\partial \Omega \times(0, \infty)$. Take $\alpha=2 s$ and $X_{0}^{\alpha}\left(\mathscr{C}_{\Omega}\right)$ as the completion of $C_{0}^{\infty}(\Omega \times(0, \infty))$ with respect to the norm

$$
\|z\|_{X_{0}^{\alpha}\left(\mathscr{C}_{\Omega}\right)}=\left(\kappa_{\alpha} \int_{\mathscr{C}_{\Omega}} y^{1-\alpha}|\nabla z|^{2} d x d y\right)^{1 / 2}
$$

where $\kappa$ is a normalization constant. For $w \in X_{0}^{\alpha}\left(\mathscr{C}_{\Omega}\right)$, let

$$
\begin{aligned}
L_{\alpha} w & :=-\operatorname{div}\left(y^{1-\alpha} \nabla w\right), \\
\frac{\partial w}{\partial \nu^{\alpha}} & :=\kappa_{\alpha} \lim _{y \rightarrow 0^{+}} y^{1-\alpha} \frac{\partial w}{\partial y}
\end{aligned}
$$


and consider the problem

$$
\begin{aligned}
L_{\alpha} w & =0 \quad \text { in } \mathscr{C}_{\Omega}, \\
w & =0 \quad \text { in } \partial_{L} \mathscr{C}_{\Omega}, \\
\frac{\partial w}{\partial \nu^{\alpha}} & =f(x, w) \quad \text { in } \Omega \times\{y=0\} .
\end{aligned}
$$

An energy solution to this problem is a function $w \in X_{0}^{\alpha}\left(\mathscr{C}_{\Omega}\right)$ such that

$$
\begin{array}{r}
\kappa_{\alpha} \int_{\mathscr{C}_{\Omega}} y^{1-\alpha}\langle\nabla w, \nabla \varphi\rangle d x d y=\int_{\Omega} f(x, w) \varphi d x \\
\forall \varphi \in X_{0}^{\alpha}\left(\mathscr{C}_{\Omega}\right) .
\end{array}
$$

Such an energy solution $w$ yields a function $u=w(\cdot, 0)$ in the sense of traces, which belongs to the space $H_{0}^{\alpha / 2}(\Omega)$ and is a weak solution of (3). The converse is also true. The reader may refer to [2-6] for dealing with (3) with this method. In particular, Stinga and Torrea [6] generalized the arguments and results in [2] to the fractional powers $L^{\sigma}, 0<\sigma<1$, of a linear second order partial differential operator $L$ that is nonnegative, densely defined, and self-adjoint in $L^{2}(\Omega, d \eta)$ with a positive measure $d \eta$ on $\Omega$.

Servadei and Valdinoci developed a variational framework to study the problem (2) in a series of papers [7-11]. They introduced the following Hilbert space $\left(X_{0}(\Omega, K),\langle\cdot, \cdot\rangle_{0, \Omega, K}\right)$ in $[7,8]$. Let $Q:=\mathbb{R}^{2 n} \backslash(\mathscr{C} \Omega \times \mathscr{C} \Omega)$, where $\mathscr{C} \Omega=\mathbb{R}^{n} \backslash \Omega$, and let $X(\Omega, K)$ be the space of all Lebesgue measurable functions $u: \mathbb{R}^{n} \rightarrow \mathbb{R}$ such that $\left.u\right|_{\Omega} \in L^{2}(\Omega)$ and that the map

$$
\begin{aligned}
& Q \ni(x, y) \\
& \qquad(u(x)-u(y)) \sqrt{K(x-y)} \text { is in } L^{2}(Q) .
\end{aligned}
$$

$X(\Omega, K)$ is a Banach space endowed with the so-called Gagliardo norm

$$
\begin{aligned}
& \|u\|_{\Omega, K}=\left(\int_{\Omega}|u(x)|^{2} d x\right. \\
& \left.\quad+\int_{Q}|u(x)-u(y)|^{2} K(x-y) d x d y\right)^{1 / 2}
\end{aligned}
$$

and is contained in $H^{s}\left(\mathbb{R}^{n}\right)$. Consider the subspace of $X(\Omega, K)$ :

$$
X_{0}(\Omega, K)=\left\{u \in X(\Omega, K) \mid u=0 \text { a.e. in } \mathbb{R}^{n} \backslash \Omega\right\} .
$$

It was proved in [12, Theorem 6] that this space is the closure of $C_{0}^{\infty}(\Omega)$ in $X(\Omega, K)$. Clearly, the space $X_{0}(\Omega, K)$ depends on $K$. In fact, when $K(x)=|x|^{-(n+2 s)}, X_{0}(\Omega, K)=\left\{u \in H^{s}\left(\mathbb{R}^{n}\right) \mid\right.$ $u=0$ a.e. in $\left.\mathbb{R}^{n} \backslash \Omega\right\}$ ([11, Lemma 7-b]). $X_{0}(\Omega, K)$ can be endowed with a Hilbert space structure given by the inner product

$$
\begin{aligned}
& \langle u, v\rangle_{0, \Omega, K} \\
& =\int_{Q}(u(x)-u(y))(v(x)-v(y)) K(x-y) d x d y
\end{aligned}
$$

([8, Lemma 7]) and contains $C_{0}^{2}(\Omega)([7$, Lemma 11]). Denote by $\|\cdot\|_{0, \Omega, K}$ the induced norm of the inner product in (10). This norm is equivalent to the restriction of $\|\cdot\|_{\Omega, K}$ to $X_{0}(\Omega, K)$. Call $u \in X_{0}(\Omega, K)$ a weak solution of the problem (2) if $u$ satisfies

$$
\begin{aligned}
\int_{\mathbb{R}^{2 n}} & (u(x)-u(y))(\phi(x)-\phi(y)) K(x-y) d x d y \\
= & \int_{\Omega} f(x, u(x)) \phi(x) d x
\end{aligned}
$$

for all $\phi \in X_{0}(\Omega, K)$. Define $F(x, t)=\int_{0}^{t} f(x, \tau) d \tau$ for the above Carathéodory function $f$. Suppose that there exist $a_{1}>$ $0, a_{2}>0$ and $q \in\left(2,2_{s}^{*}\right), 2_{s}^{*}=2 n /(n-2 s)$, such that

$$
|f(x, t)| \leq a_{1}+a_{2}|t|^{q-1} \quad \text { a.e. } x \in \Omega, t \in \mathbb{R} .
$$

Then, the functional $\mathscr{J}: X_{0}(\Omega, K) \rightarrow \mathbb{R}$ defined by

$$
\begin{aligned}
\mathscr{J}(u)= & \frac{1}{2} \int_{Q}|u(x)-u(y)|^{2} K(x-y) d x d y \\
& -\int_{\Omega} F(x, u(x)) d x
\end{aligned}
$$

is of class $C^{1}$ and the critical points of $\mathscr{J}$ are exactly the weak solutions of (2). Condition (12) is always assumed in the proofs of present several existence results on (2) via variational methods [7-11, 13-15]. Except for [13] $u=0$ is also assumed to be a solution in all other works. For studies of (3), there is a great deal of literature; see $[2,4,16]$ and references therein.

However, all previous results cannot include the following case: $\Omega=B^{n}(0,1)=\left\{x \in \mathbb{R}^{n}|| x \mid<1\right\}$ and $f(x, t)=$ $1 /(|x|-1)+h(t)$, where $h \in C^{1}(\mathbb{R})$ such that $h(t)=\cos t$ for $|t| \ll 1$ and that $h(t)=\sin t$ for $|t| \gg 1$.

1.2. Main Results of This Paper. We will use the topological degree theory developed by [17] to study generalized solutions of problem (2).

Our result can apply to the example just mentioned. Without special statements, we write

$$
\begin{aligned}
r & =\frac{2 n}{(n+2 s)}, \\
2_{s}^{*} & =\frac{2 n}{(n-2 s)}
\end{aligned}
$$

(the latter plays the role of a critical Sobolev exponent).

Theorem 1. For an integer $n \geq 2$ let $K: \mathbb{R}^{n} \backslash\{0\} \rightarrow(0,+\infty)$ satisfy (i)-(iii) and let $f: \Omega \times \mathbb{R} \rightarrow \mathbb{R}$ be a Carathéodory function verifying the following conditions: there exist positive numbers $\alpha, \beta, q \in\left(2,2_{s}^{*}\right), p \in[1,2)$ and functions $a \in L_{\mathrm{loc}}^{r}(\Omega)$ and $c \in L^{1}(\Omega)$ such that

$$
\begin{gathered}
|f(x, t)| \leq a(x)+\alpha|t|^{q-1} \quad \forall(x, t) \in \Omega \times \mathbb{R}, \\
\Omega \ni x \longmapsto b(x) \\
:=f(x, 0) \text { belongs to } L^{r}(\Omega), \\
-f(x, t) t \geq-\beta|t|^{p}-c(x) \quad \forall(x, t) \in \Omega \times \mathbb{R} .
\end{gathered}
$$


Then, problem (2) has at least one generalized solution $u$ in $X_{0}(\Omega, K)$; that is, it satisfies

$$
\begin{gathered}
\int_{\mathbb{R}^{2 n}}(u(x)-u(y))(\phi(x)-\phi(y)) K(x-y) d x d y \\
=\int_{\Omega} f(x, u(x)) \phi(x) d x \quad \forall \phi \in C_{0}^{\infty}(\Omega) .
\end{gathered}
$$

In particular, it must have a nontrivial generalized solution if $b$ is not identically zero.

Corollary 2. Under the assumptions of Theorem 1, let $1<v<$ $2, l>2 n /(2 n-(n-2 s) \nu)$ and let $G \in L_{\mathrm{loc}}^{l}(\Omega)$. If either $\lambda G \leq 0$ or $(\lambda G)^{+}:=\max \{0, \lambda G\}$ belongs to $L^{\kappa}(\Omega)$ with $\kappa>2 /(2-\nu)$, then

$$
\begin{aligned}
\mathfrak{\Omega}_{K} u+\lambda G(x)|u|^{\nu-2} u+f(x, u) & =0 \quad \text { in } \Omega, \\
u & =0 \text { in } \mathbb{R}^{n} \backslash \Omega,
\end{aligned}
$$

has at least one nontrivial solution in $X_{0}(\Omega, K)$ provided $b$ is not identically zero.

Corollary 3. Under the assumptions of Theorem 1 , let $2 \leq v<$ $2_{s}^{*}$ and $G \in L_{\mathrm{loc}}^{l}(\Omega)$ with $l \geq r \vartheta$ and $\vartheta>(n+2 s) /(n+2 s-(\nu-$ 1) $(n-2 s))$. If $\lambda \in \mathbb{R}$ is such that $\lambda G \leq 0$, then

$$
\begin{aligned}
\mathfrak{R}_{K} u+\lambda G(x)|u|^{\nu-2} u+f(x, u) & =0 \text { in } \Omega, \\
u & =0 \text { in } \mathbb{R}^{n} \backslash \Omega,
\end{aligned}
$$

has at least one nontrivial solution in $X_{0}(\Omega, K)$ provided $b$ is not identically zero.

In particular, this corollary includes the example at the end of Section 1.2. See Example 1 for more general cases.

Our methods can also be used to study the case of nonlocal elliptic operator systems. Let $K_{1}, K_{2}: \mathbb{R}^{n} \backslash\{0\} \rightarrow$ $(0,+\infty)$ be functions satisfying conditions (i)-(iii). Given two Carathéodory functions $f_{i}: \Omega \times \mathbb{R} \rightarrow \mathbb{R}, i=1,2$, consider the following problem:

$$
\begin{aligned}
\mathfrak{\Omega}_{K_{1}} u+f_{1}(x, v)=0 & \text { in } \Omega, \\
\mathfrak{\Omega}_{K_{2}} v+f_{2}(x, u)=0 & \text { in } \Omega, \\
u=v=0 & \text { in } \mathbb{R}^{n} \backslash \Omega .
\end{aligned}
$$

Call $(u, v) \in X_{0}\left(\Omega, K_{1}\right) \times X_{0}\left(\Omega, K_{2}\right)$ a generalized solution of system (21) if

$$
\begin{aligned}
& \int_{\mathbb{R}^{2 n}}(u(x)-u(y))(\phi(x)-\phi(y)) K_{1}(x-y) d x d y \\
& +\int_{\mathbb{R}^{2 n}}(v(x)-v(y))(\psi(x)-\psi(y)) \\
& \cdot K_{2}(x-y) d x d y-\int_{\Omega} f_{1}(x, v) \phi(x) d x \\
& -\int_{\Omega} f_{2}(x, u) \psi(x) d x=0
\end{aligned}
$$

for every $(\phi, \psi) \in C_{0}^{\infty}(\Omega) \times C_{0}^{\infty}(\Omega)$. Here is the second main result.
Theorem 4. Under the above assumptions, suppose also that there exist positive numbers $\alpha_{j}, \beta_{j}, q_{j} \in\left(2,2_{s}^{*}\right), 1 \leq p_{j}, \widehat{p}_{j}<2$, and functions $a_{j} \in L_{\mathrm{loc}}^{r}(\Omega)$ and $c_{j} \in L^{1}(\Omega)$ such that

$$
\begin{aligned}
& \left|f_{j}(x, t)\right| \leq a_{j}(x)+\alpha_{i}|t|^{q_{j}-1} \quad \forall(x, t) \in \Omega \times \mathbb{R}, \\
& \Omega \ni x \longmapsto b_{j}(x) \\
& :=f_{j}(x, 0) \text { belongs to } L^{r}(\Omega), \\
& -f_{j}(x, t) h \geq-\beta_{j}|t|^{p_{j} / 2}|h|^{\widehat{p}_{j} / 2}-c_{j}(x) \\
& \forall(x, t, h) \in \Omega \times \mathbb{R} \times \mathbb{R} .
\end{aligned}
$$

Then, problem (21) has at least one generalized solution in $X_{0}\left(\Omega, K_{1}\right) \times X_{0}\left(\Omega, K_{2}\right)$. In particular, it must have a nontrivial generalized solution if one of $b_{1}$ and $b_{2}$ is not identically zero.

Similarly, consider the following problem:

$$
\begin{aligned}
& -\Delta u(x)+\sum_{j=1}^{n} f_{j}(x, v(x)) \frac{\partial u}{\partial x_{j}}(x)+f_{0}(x, v(x)) \\
& +a_{1}(x)=0, \\
& -\Delta v(x)+\sum_{j=1}^{n} g_{j}(x, u(x)) \frac{\partial v}{\partial x_{j}}(x)+g_{0}(x, u(x)) \\
& +a_{2}(x)=0
\end{aligned}
$$

or

$$
\begin{aligned}
& -\Delta u(x)+\sum_{j=1}^{n} f_{j}(x, v(x)) \frac{\partial v}{\partial x_{j}}(x)+f_{0}(x, v(x)) \\
& +a_{1}(x)=0, \\
& -\Delta v(x)+\sum_{j=1}^{n} g_{j}(x, u(x)) \frac{\partial u}{\partial x_{j}}(x)+g_{0}(x, u(x)) \\
& +a_{2}(x)=0 .
\end{aligned}
$$

Assume

(A) $f_{i}, g_{i}: \Omega \times \mathbb{R}^{n} \rightarrow \mathbb{R}$ are Carathéodory functions, $i=0, \ldots, n$;

(B) there exist constants

$$
\begin{aligned}
& r_{0} \in\left(\frac{2 n}{n+2}, \infty\right), \\
& r_{i} \in(n, \infty), \quad \frac{n-2}{2 n} r_{i}<\frac{1}{s_{i}}<\infty, i=1, \ldots, n,
\end{aligned}
$$


and measurable functions $b_{i}, d_{i} \in L_{\text {loc }}^{r_{i}}(\Omega), i=$ $0,1, \ldots, n, a_{1}, a_{2} \in L^{r_{0}}(\Omega)$, such that

$$
\begin{aligned}
& f_{0}(x, 0)=g_{0}(x, 0)=0, \quad \forall x \in \Omega, \\
&\left|f_{i}(x, t)\right| \leq b_{i}(x)+k_{i}|t|^{s_{i}}, \\
& \forall(x, t) \in \Omega \times \mathbb{R}, i=0, \ldots, n, \\
&\left|g_{i}(x, t)\right| \leq d_{i}(x)+l_{i}|t|^{s_{i}}, \\
& \forall(x, t) \in \Omega \times \mathbb{R}, i=0, \ldots, n ;
\end{aligned}
$$

(C) there exist measurable functions $c(x), \widehat{c}(x) \in L^{1}(\Omega)$ and constants $1<q_{1}, q_{2}, \widehat{q}_{1}, \widehat{q}_{2}<2$ such that, for any $\left(x, t_{1}, t_{2}, z\right) \in \Omega \times \mathbb{R} \times \mathbb{R} \times \mathbb{R}^{n}$,

$$
\begin{aligned}
& -\frac{1}{2}|z|^{2}-k\left|t_{1}\right|^{q_{1} / 2}\left|t_{2}\right|^{\widehat{q}_{1} / 2} c(x) \\
& \leq\left[\sum_{i=1}^{n} f_{i}\left(x, t_{2}\right) z_{i}+f_{0}\left(x, t_{2}\right)+a_{1}(x)\right] t_{1}, \\
& -\frac{1}{2}|z|^{2}-\widehat{k}\left|t_{1}\right|^{q_{2} / 2}\left|t_{2}\right|^{\widehat{q}_{2} / 2} \widehat{c}(x) \\
& \leq\left[\sum_{i=1}^{n} g_{i}\left(x, t_{2}\right) z_{i}+g_{0}\left(x, t_{2}\right)+a_{2}(x)\right] t_{1} .
\end{aligned}
$$

Combing the proof of [17] and that of Theorem 4, we can prove the following.

Theorem 5. Under the conditions (A), (B), and (C), if $a_{1}$ or $a_{2}$ is not zero, then the equation systems (26) and (27) have at least a nontrivial generalized solution $(u, v) \in W_{0}^{1,2}(\Omega) \times W_{0}^{1,2}(\Omega)$.

Finally, let us point out that the corresponding results of Theorems 1 and 4 can be also proved if the operator $\mathfrak{R}_{K}$ is replaced by $L^{\sigma}$ in $[6,(1.10)]$. They will be given in other places.

The arrangements of this paper are as follows. In Section 2, we give some necessary preliminaries. The proof of Theorem 1 will be completed in Section 3. In Section 4, we will prove Corollaries 2 and 3 and give an example. Theorem 4 will be proved in Section 5 .

\section{Preliminaries}

Firstly, we review the topological degree theory for mappings of class $\left(B_{+}\right)$developed in [17]. Let $H$ be a Hilbert space with inner product $\langle\cdot, \cdot\rangle$ and let $\left\{E_{n}\right\}_{n}$ be a strictly increasing sequence of finite dimensional subspace of $H$ such that $E=$ $\cup_{n=1}^{\infty} E_{n}$ is dense in $H$. Denote by $P_{n}$ the orthogonal projection from $H$ onto $E_{n}$ for every integer $n \in \mathbb{N}$. Let $G$ be an open bounded set in $E$ and let $g$ be a mapping from $\bar{G}^{E}$, the closure of $G$ in $E$, into $H$. Put

$$
\begin{aligned}
G_{n} & =G \cap E_{n} \quad \forall n \in \mathbb{N}, \\
g_{n}(x) & =P_{n}(g(x)) \quad \forall n \in \mathbb{N}, x \in \overline{G_{n} .}
\end{aligned}
$$

Since $H$ and $E$ induce equivalent topologies on all finite dimensional spaces $E_{n}$ the subset $G_{n} \subset E_{n}$ has the same closure in $E_{n}, E$, and $H$, denoted by $\frac{G_{n}}{G_{n}}$.

Definition 6. Under the above assumptions, $g$ is said to be of class $\left(B_{+}\right)$on $\bar{G}^{E}$ if and only if the following conditions are satisfied:

(a) $g_{n}: \overline{G_{n}} \rightarrow E_{n}$ is a continuous mapping for each $n \epsilon$ $\mathbb{N}$.

(b) There is not any sequence $\left\{x_{n_{k}}\right\}_{k}$ in $E$ such that the sequence $\left\{x_{n_{k}}\right\}_{k}$ is weakly convergent in $H, x_{n_{k}} \in$ $\partial_{E_{n_{k}}} G_{n_{k}},\left\langle g\left(x_{n_{k+1}}\right), x_{n_{k+1}}\right\rangle \leq 0$ and $\left\langle g\left(x_{n_{k+1}}\right), v\right\rangle=0$ for all $k \in \mathbb{N}$ and $v$ in $E_{n_{k}}$.

Lemma 7 (see [17, Lemma 2.3]). Let $H,\left\{E_{n}\right\}_{n}, E, G, g$ and $\left\{g_{n}\right\}_{n}$ be as in Definition 6. Assume that $g$ is of class $\left(B_{+}\right)$on $\bar{G}^{E}$. Then, there exists an integer $n_{0}$ such that the Leray-Schauder degree $\operatorname{deg}\left(g_{n}, G_{n}, 0\right)$ is defined and

$$
\operatorname{deg}\left(g_{n}, G_{n}, 0\right)=\operatorname{deg}\left(g_{n_{0}}, G_{n_{0}}, 0\right) \quad \forall n \geq n_{0} .
$$

It follows that

$$
\operatorname{deg}(g, G, 0):=\lim _{n \rightarrow \infty} \operatorname{deg}\left(g_{n}, G_{n}, 0\right)
$$

is defined. It was the topological degree of $g$ on $G$ at 0 in [17]. The corresponding versions with usual properties of the Leray-Schauder degree were given in [17, Theorem 2.1]. In particular, the identity map Id is of class $\left(B_{+}\right)$, and $\operatorname{deg}(\mathrm{Id}, G, 0)=1$ if $0 \in G$. Moreover, the following proposition is key for the proof of our main results.

Proposition 8 (see [17, Corollary 2.1]). Let $H,\left\{E_{n}\right\}_{n}, E$ and $G$ be as in Definition 6. Let $g$ be a mapping from $\bar{G}^{E}$ into $H$ such that $g_{m}$ is continuous on $\bar{G}_{m} E_{m}$ for any $m \in \mathbb{N}$. Suppose that $G$ contains 0 and

$$
\langle g(x), x\rangle>0, \quad \forall x \in \partial_{E} G .
$$

Then there is a weakly Cauchy sequence $\left\{x_{n}\right\}_{n}$ in G such that

$$
\lim _{n \rightarrow \infty}\left\langle g\left(x_{n}\right), v\right\rangle=0, \quad \forall v \in E .
$$

Next, we need the following results on the space $X_{0}(\Omega, K)$.

Lemma 9. (a) $X_{0}(\Omega, K)$ and $X(\Omega, K)$ are continuously embedded in $H^{s}\left(\mathbb{R}^{n}\right)$ and $H^{s}(\Omega)$, respectively $([8$, Lemma 5$])$.

(b) If $\Omega \subset \mathbb{R}^{n}$ is a bounded open subset with continuous boundary, the embedding $X_{0}(\Omega, K) \hookrightarrow L^{p}\left(\mathbb{R}^{n}\right)$ is compact for any $p \in\left[1,2_{s}^{*}\right)([8$, Lemma 8$]$ and $[11$, Lemma 9-a]).

(c) The embedding $X_{0}(\Omega, K) \hookrightarrow L^{p}\left(\mathbb{R}^{n}\right)$ is continuous for $p=2_{s}^{*}([11$, Lemma 9-b]).

(d) The embedding $H^{s}\left(\mathbb{R}^{n}\right) \hookrightarrow L^{p}\left(\mathbb{R}^{n}\right)$ is continuous for any $p \in\left[1,2_{s}^{*}\right]([18$, Theorem 6.5$])$.

(e) If $\Omega$ is an open set in $\mathbb{R}^{n}$ of class $C^{0,1}$ with bounded boundary, then there exist continuous embeddings $W^{1, p}(\Omega) \hookrightarrow$ $W^{s, p}(\Omega)$ and $W_{0}^{1, p}(\Omega) \hookrightarrow W_{0}^{s, p}(\Omega)$ for any $p \in[1, \infty)$ and $s \in(0,1)([18$, Proposition 2.2]). 
Lemma 10. Let $\Omega$ be a bounded open set in $\mathbb{R}^{n}$ with boundary of class $C^{0,1}$. Then, the space $X_{0}(\Omega, K)$ is separable. Furthermore, there exists a sequence $\left\{v_{m}\right\}_{m}$ in $C_{0}^{\infty}(\Omega)$ such that $\left\{v_{m}\right\}_{m}$ is a maximal orthogonal set of $X_{0}(\Omega, K)$.

Proof. By Proposition 9(f) of [9], there exists a Hilbert basis $\left\{e_{k}\right\}_{k \geq 1}$ of $X_{0}(\Omega, K)$, which implies separability of $X_{0}(\Omega, K)$. So $\cup_{m=1}^{\infty}\left\{r_{1} e_{1}+\cdots+r_{m} e_{m} \mid r_{i} \in \mathbb{Q}, i=1, \ldots, m\right\}$ is a dense countable subset in $X_{0}(\Omega, K)$. Let $\left\{f_{m}\right\}_{m>1}$ denote this countable set. Since $C_{0}^{\infty}(\Omega)$ is dense in $X_{0}(\Omega, K)$ by [12, Theorem 6], for each $m \in \mathbb{N}$ we can take $f_{m, k} \in C_{0}^{\infty}(\Omega)$ such that $\left\|f_{m}-f_{m, k}\right\|_{0, \Omega, K}<1 / k \forall k \in \mathbb{N}$. Then, $\left\{f_{m, k} \mid\right.$ $(m, k) \in \mathbb{N} \times \mathbb{N}\}$ is also dense in $X_{0}(\Omega, K)$. Let $\left\{h_{l} \mid l \in \mathbb{N}\right\}$ be a maximal subset of $\left\{f_{m, k} \mid(m, k) \in \mathbb{N} \times \mathbb{N}\right\}$ such that any finite elements in $\left\{h_{l} \mid l \in \mathbb{N}\right\}$ are linearly independent. Then, $\operatorname{Span}\left(\left\{h_{l} \mid l \in \mathbb{N}\right\}\right)=\operatorname{Span}\left(\left\{f_{m, k} \mid(m, k) \in \mathbb{N} \times \mathbb{N}\right\}\right)$ is dense in $X_{0}(\Omega, K)$. Making the Hilbert-Schmidt orthogonalization procedure for $\left\{h_{m} \mid m \in \mathbb{N}\right\}$, we obtain an orthogonal set $\left\{e_{m} \mid m \in \mathbb{N}\right\}$, which is also a maximal in $X_{0}(\Omega, K)$.

\section{Proof of Theorem 1}

Take an increasing sequence of open subsets of $\Omega,\left\{\Omega_{k}\right\}_{k}$, such that each of them has $C^{0,1}$-boundary and that

$$
\begin{aligned}
& \overline{\Omega_{k}} \subset \Omega_{k+1} \quad \forall k \in \mathbb{N}, \\
& \Omega=\bigcup_{k=1}^{\infty} \Omega_{k} .
\end{aligned}
$$

By Lemma 10, we may choose a sequence $\left\{v_{1, m}\right\}_{m}$ in $C_{0}^{\infty}\left(\Omega_{1}\right)$ such that $\left\{v_{1, m}\right\}_{m}$ is a maximal orthogonal set of $X_{0}\left(\Omega_{1}, K\right)$. Then, we can find a sequence $\left\{v_{2, m}\right\}_{m}$ in $C_{0}^{\infty}\left(\Omega_{2}\right) \backslash C_{0}^{\infty}\left(\Omega_{1}, K\right)$ such that $\left\{v_{1, m}, v_{2, m} \mid m \in \mathbb{N}\right\}$ is a maximal orthogonal set of $X_{0}\left(\Omega_{2}, K\right)$. By the mathematical induction, it is easy to find the set $\left\{v_{k, m} \mid k, m \in \mathbb{N}\right\}$ in $C_{0}^{\infty}(\Omega)$ such that $\left\{v_{j, m} \mid m \in \mathbb{N}, j=\right.$ $1, \ldots, k\}$ is a maximal orthogonal set of $X_{0}\left(\Omega_{k}, K\right)$ for every $k \in \mathbb{N}$. Let us rewrite the countable set $\left\{v_{k, m} \mid k, m \in \mathbb{N}\right\}$ as a sequence $\left\{e_{k}\right\}_{k}$. Let $E_{m}$ be the vector subspace of $X_{0}(\Omega, K)$ spanned by $\left\{e_{1}, \ldots, e_{m}\right\}$, and $E=\cup_{m} E_{m}$. For conveniences we set $H=X_{0}(\Omega, K)$ and denote by $P_{m}$ the orthogonal projection from $H$ onto $E_{m}$.

Lemma 11. (a) $E$ is dense in $X_{0}(\Omega, K)$.

(b) For each $u \in C_{0}^{\infty}(\Omega)$, there are $k \in \mathbb{N}$ and a sequence $\left\{u_{m}\right\}_{m}$ in E such that the supports of all $u_{m}$ are contained in $\Omega_{k}$ and that $u_{m} \rightarrow u$ in $X_{0}(\Omega, K)$ as $m \rightarrow \infty$.

(c) For every $m \in \mathbb{N}$ and for every given $u \in X_{0}(\Omega, K)$, there exists a unique $T_{m}(u)$ in $X_{0}(\Omega, K)$ such that

$$
\left\langle T_{m}(u), v\right\rangle_{0, \Omega, K}=-\int_{\Omega_{m}} f(x, u(x)) v(x) d x
$$

$$
\forall v \in X_{0}(\Omega, K) \text {. }
$$

Moreover, if $v \in C_{0}^{\infty}\left(\Omega_{k}\right)$, then

$$
\left\langle T_{m}(u), v\right\rangle_{0, \Omega, K}=\left\langle T_{k}(u), v\right\rangle_{0, \Omega, K} \quad \forall m \geq k .
$$

(d) Suppose that a sequence $\left\{u_{k}\right\}_{k} \subset X_{0}(\Omega, K)$ weakly converges to $u$ in $X_{0}(\Omega, K)$. Then, $\left\{T_{m}\left(u_{k}\right)\right\}_{k}$ weakly converges to $T_{m}(u)$ in $X_{0}(\Omega, K)$ for $m=1,2, \ldots$. (e) For every given $u \in E$ (the support of $u$ must be contained in some $\Omega_{m_{0}}$ by the construction of $E$ ), there exists a unique $T(u) \in X_{0}(\Omega, K)$ such that

$$
\begin{aligned}
&\langle T(u), v\rangle_{0, \Omega, K}=-\int_{\Omega} f(x, u(x)) v(x) d x \\
& \forall v \in X_{0}(\Omega, K), \\
&\langle T(u), v\rangle_{0, \Omega, K}=\left\langle T_{m}(u), v\right\rangle_{0, \Omega, K} \\
& \forall v \in X_{0}(\Omega, K), \quad \forall m \geq m_{0} .
\end{aligned}
$$

(f) $P_{m} \circ\left(\left.T\right|_{E_{m}}\right): E_{m} \rightarrow E_{m}$ is continuous for every $m \in \mathbb{N}$. (g) There exists a constant $C>0$ such that

$$
\begin{aligned}
& \langle u+T(u), u\rangle_{0, \Omega, K} \\
& \quad \geq\|u\|_{0, \Omega, K}^{2}\left(1-C\|u\|_{0, \Omega, K}^{p-2}-\|c\|_{L^{1}(\Omega)}\|u\|_{0, \Omega, K}^{-2}\right)
\end{aligned}
$$$$
\forall u \in E \backslash\{0\} .
$$

Proof. (a) Since $\left\{e_{k}\right\}_{k}$ is a maximal orthogonal set of $X_{0}(\Omega, K), E$ is dense in $X_{0}(\Omega, K)$.

(b) For a given $u \in C_{0}^{\infty}(\Omega)$, by the choices of $\left\{\Omega_{m}\right\}_{m}$, there exists $k \in \mathbb{N}$ such that the support of $u$ is contained in $\Omega_{k}$. Let $\left\{v_{j, m} \mid m \in \mathbb{N}, j=1, \ldots, k\right\}$ be the maximal orthogonal set of $X_{0}\left(\Omega_{k}, K\right)$ as constructed above. Then, $\operatorname{Span}\left(\left\{v_{j, m} \mid m \in\right.\right.$ $\mathbb{N}, j=1, \ldots, k\})$ is dense in $X_{0}\left(\Omega_{k}, K\right)$. Hence, we can find a sequence $\left\{u_{m}\right\}_{m \geq 1}$ in $E \cap C_{0}^{\infty}\left(\Omega_{k}\right)$ such that $\left\|u_{m}-u\right\|_{0, \Omega, K} \rightarrow 0$ as $m \rightarrow \infty$.

(c) By (16), we can write $f(x, t)=b(x)+f_{0}(x, t) \forall(x, t) \epsilon$ $\Omega \times \mathbb{R}$. Then, $f_{0}(x, 0)=0 \forall x \in \Omega$, and (15) implies

$$
\left|f_{0}(x, t)\right| \leq a(x)+b(x)+\alpha|t|^{q-1} \quad \forall(x, t) \in \Omega \times \mathbb{R} .
$$

Note that $2_{s}^{*}<2 n /(n-2)$ and $1 / r+1 / 2_{s}^{*}=1$. Moreover, since $q \in\left(2,2_{s}^{*}\right)$ we get

$$
\begin{aligned}
\frac{2 n}{n+2 s} & =r<r(q-1)<r\left(2_{s}^{*}-1\right) \\
& =\frac{2 n}{n+2 s}\left(\frac{2 n}{n-2 s}-1\right)=\frac{2 n}{n-2 s}=2_{s}^{*} .
\end{aligned}
$$

For given $m \in \mathbb{N}$, by [19, Theorem 3.2.4] (see also [20, page $30]$ ) we have a continuous mapping $\mathfrak{F}_{m}$ from $L^{r(q-1)}(\Omega)$ into $L^{r}\left(\Omega_{m}\right)$, where

$$
\mathfrak{\Im}_{m}(u)(x)=-f_{0}(x, u(x)) \quad \forall x \in \Omega_{m} .
$$

For $u, v \in X_{0}(\Omega, K)$, we have $u \in L^{r(q-1)}(\Omega)$ and $v \in L^{2_{s}^{*}}(\Omega)$ by Lemma 9 (b) and (c). Thus, $\mathfrak{F}_{m}(u) \in L^{r}\left(\Omega_{m}\right)$ and

$$
\begin{aligned}
& \left|\int_{\Omega_{m}} f_{0}(x, u(x)) v(x) d x\right| \\
& \quad=\left|\int_{\Omega_{m}} \mathfrak{F}_{m}(u) v(x) d x\right| \leq\left\|\mathfrak{F}_{m}(u)\right\|_{L^{r}\left(\Omega_{m}\right)}\|v\|_{L^{2_{s}^{*}}} .
\end{aligned}
$$


Using Lemma 9(c) again, there exists a constant $C>0$ such that $\|v\|_{L^{2 *}} \leq C\|v\|_{0, \Omega, K}$ for all $v \in X_{0}(\Omega, K)$. It follows that

$$
\begin{gathered}
\left|-\int_{\Omega_{m}} f_{0}(x, u(x)) v(x) d x-\int_{\Omega} b(x) v(x) d x\right| \\
\leq C\left(\left\|\mathfrak{\Im}_{m}(u)\right\|_{L^{r}\left(\Omega_{m}\right)}+\|b\|_{L^{r}(\Omega)}\right)\|v\|_{0, \Omega, K} .
\end{gathered}
$$

Hence, Riesz representative theorem yields a unique $T_{m}(u) \epsilon$ $X_{0}(\Omega, K)$ such that

$$
\begin{array}{r}
\left\langle T_{m}(u), v\right\rangle_{0, \Omega, K}=\int_{\Omega_{m}} \mathfrak{F}_{m}(u) v d x-\int_{\Omega} b(x) v(x) \\
\forall v \in X_{0}(\Omega, K) .
\end{array}
$$

If $v \in C_{0}^{\infty}\left(\Omega_{k}\right)$, for each integer $m \geq k$ we deduce

$$
\begin{aligned}
\left\langle T_{m}(u), v\right\rangle_{0, \Omega, K} & =\int_{\Omega_{m}} \mathfrak{F}_{m}(u) v d x-\int_{\Omega} b(x) v(x) \\
& =\int_{\Omega_{k}} \mathfrak{F}_{k}(u) v d x-\int_{\Omega} b(x) v(x) \\
& =\left\langle T_{k}(u), v\right\rangle_{0, \Omega, K} .
\end{aligned}
$$

(d) Let $\left\{u_{k}\right\}_{k}$ be a sequence weakly converging to $u$ in $X_{0}(\Omega, K)$. Since

$$
1<r(q-1)<2_{s}^{*}<\frac{2 n}{n-2},
$$

from Lemma 9(b), we deduce that $\left\{u_{k}\right\}_{k}$ converges to $u$ in $L^{r(q-1)}(\Omega)$. Then, the continuity of the map $\mathfrak{F}_{m}$ : $L^{r(q-1)}(\Omega) \rightarrow L^{r}\left(\Omega_{m}\right)$ implies that $\left\{\mathfrak{F}_{m}\left(u_{k}\right)\right\}_{k}$ converges to $\mathfrak{F}_{m}(u)$ in $L^{r}\left(\Omega_{m}\right)$. Moreover, for $v \in X_{0}(\Omega, K)$, we have $v \in$ $L^{2_{s}^{*}}(\Omega)$. Recall that $r^{-1}+\left(2_{s}^{*}\right)^{-1}=1$ and $L^{2_{s}^{*}}(\Omega)=\left(L^{r}(\Omega)\right)^{*}$. We deduce that

$$
\begin{array}{r}
\int_{\Omega_{m}} v(x) \mathfrak{\mho}_{m}\left(u_{k}\right)(x) d x \longrightarrow \int_{\Omega_{m}} v(x) \mathfrak{F}_{m}(u)(x) d x \\
\text { as } k \longrightarrow \infty,
\end{array}
$$

and hence $\lim _{k \rightarrow \infty}\left\langle T_{m}\left(u_{k}\right), v\right\rangle_{0, \Omega, K}=\left\langle T_{m}(u), v\right\rangle_{0, \Omega, K}$ by $(46)$.

(e) Since $f_{0}(x, 0)=0 \forall x \in \Omega$, and $\operatorname{Supp}(u) \subset \Omega_{m_{0}}$, by (46) we deduce that

$$
\begin{aligned}
& -\int_{\Omega} f(x, u(x)) v(x) d x \\
& =-\int_{\Omega} f_{0}(x, u(x)) v(x) d x-\int_{\Omega} b(x) v(x) d x \\
& =-\int_{\Omega_{m}} f_{0}(x, u(x)) v(x) d x-\int_{\Omega} b(x) v(x) d x \\
& =\left\langle T_{m}(u), v\right\rangle_{0, \Omega, K} \quad \forall v \in X_{0}, \quad \forall m \geq m_{0} .
\end{aligned}
$$

This shows that $X_{0}(\Omega, K) \ni v \mapsto-\int_{\Omega} f(x, u(x)) v(x) d x$ is a continuous linear functional. Using the Riesz representative theorem again we obtain a unique $T(u) \in X_{0}(\Omega, K)$ such that

$$
\langle T(u), v\rangle_{0, \Omega, K}=-\int_{\Omega} f(x, u(x)) v(x) d x \quad \forall v \in X_{0} .
$$

Clearly, $\langle T(u), v\rangle_{0, \Omega, K}=\left\langle T_{m}(u), v\right\rangle_{0, \Omega, K} \forall v \in X_{0}$ for all $m \geq$ $m_{0}$.

(f) By the construction of $E_{m}$, we have an integer $m_{0} \in \mathbb{N}$ such that each $u \in E_{m}$ has a support contained in $\Omega_{m_{0}}$. Let $\left\{u_{k}\right\}_{k} \subset E_{m}$ converge to $u \in E_{m}$. By $(\mathrm{d}),\left\{T_{n}\left(u_{k}\right)\right\}_{k}$ weakly converges to $T_{n}(u)$ in $X_{0}(\Omega, K)$ for every $n \in \mathbb{N}$. Then, (e) implies that $\left\langle T\left(u_{k}\right), v\right\rangle_{0, \Omega, K} \rightarrow\langle T(u), v\rangle_{0, \Omega, K} \forall v \in X_{0}(\Omega, K)$ as $k \rightarrow \infty$. In particular, since $v \in E_{m}$ satisfies $P_{m} v=v$, we have

$$
\begin{aligned}
\left\langle P_{m} \circ T\left(u_{k}\right), v\right\rangle_{0, \Omega, K} & =\left\langle T\left(u_{k}\right), P_{m} v\right\rangle_{0, \Omega, K} \\
& \longrightarrow\left\langle T(u), P_{m} v\right\rangle_{0, \Omega, K} \\
& =\left\langle P_{m} \circ T(u), v\right\rangle_{X_{0, \Omega, K}}
\end{aligned}
$$

This shows that $\left\{P_{m} \circ T\left(u_{k}\right)\right\}_{k}$ weakly converges to $P_{m} T(u)$ in $E_{m}$. However, the strong converge and the weak ones on finitely dimensional space $E_{m}$ are equivalent. Hence, $P_{m}$ 。 $T\left(u_{k}\right) \rightarrow P_{m} \circ T(u)$ as $m \rightarrow \infty$.

(g) Since $1 \leq p<2<2_{s}^{*}$, by Lemma 9(b) and (c) there is a constant $C>0$ such that $\|u\|_{L^{p}(\Omega)} \leq C\|u\|_{0, \Omega, K} \forall u \in$ $X_{0}(\Omega, K)$. It follows from this and (17) that

$$
\begin{aligned}
\langle u+T(u), u\rangle_{0, \Omega, K} & =\|u\|_{0, \Omega, K}^{2} \\
& -\int_{\Omega} f(x, u(x)) u(x) d x \\
\geq & \|u\|_{0, \Omega, K}^{2}-\int_{\Omega}\left(\beta|u|^{p}+|c|\right) d x \\
& =\|u\|_{0, \Omega, K}^{2}-\beta \int_{\Omega}|u|^{p} d x-\|c\|_{L^{1}} \\
& =\|u\|_{0, \Omega, K}^{2}-\beta\|u\|_{L^{p}}^{p}-\|c\|_{L^{1}} \\
\geq & \|u\|_{0, \Omega, K}^{2}-\beta C^{p}\|u\|_{0, \Omega, K}^{p} \\
& \quad-\|c\|_{L^{1}} .
\end{aligned}
$$

This leads to (g).

Proof of Theorem 1. Let $\beta, C$, and $c$ be as above. Since $1 \leq p<$ 2 , we have $R>0$ such that

$$
1-\beta C^{p} R^{p^{-2}}-\|c\|_{L^{1}} R^{-2}>\frac{1}{4}
$$

Let $G=\left\{u \in E:\|u\|_{0, \Omega, K}<R\right\}$. Define $g: \bar{G}^{E} \rightarrow X_{0}(\Omega, K)$ by

$$
g(u)=u+T(u) \quad \forall u \in \bar{G}^{E}
$$

Let us prove that $g$ is of class $\left(B_{+}\right)$on $\bar{G}^{E}$. Note that $G_{n}=$ $G_{n} \cap E_{n}$ has the same closure in $E_{n}, E$, and $H$, denoted by $\overline{G_{n}}$. Let $g_{n}: \overline{G_{n}} \rightarrow E_{n}$ be defined by $g_{n}(u)=P_{n}(g(u))=$ $P_{n} u+P_{n} \circ T(u)=u+P_{n} \circ T(u)$ for each $n \in \mathbb{N}$. Suppose that a sequence $\left\{u_{k}\right\}_{k} \subset \overline{G_{n}}$ converges to $u \in \overline{G_{n}}$. Lemma 11(f) 
implies $P_{n} \circ T\left(u_{k}\right) \rightarrow P_{n} \circ T(u)$ in $E_{n}$. Hence, $g_{n}$ is continuous. By the proof of Lemma $11(\mathrm{~g})$ and (54) we deduce that

$$
\begin{aligned}
\langle g(u), u\rangle_{0, \Omega, K}= & \langle u+T(u), u\rangle_{0, \Omega, K} \geq \frac{R^{2}}{4} \\
& \forall u \in \partial_{E} G=\left\{u \in E:\|u\|_{0, \Omega, K}=R\right\} .
\end{aligned}
$$

This implies that Definition 6(b) is satisfied. Hence, $g$ is of class $\left(B_{+}\right)$on $\bar{G}^{E}$. Moreover, it also shows that $g$ satisfies the conditions of Proposition 8. Thus, we have a weakly Cauchy sequence $\left\{u_{n}\right\}_{n} \subset G$ such that

$$
\lim _{n \rightarrow \infty}\left\langle g\left(u_{n}\right), v\right\rangle_{0, \Omega, K}=0 \quad \forall v \in E .
$$

Let $u$ be the weak limit of $\left\{u_{n}\right\}_{n}$ in $X_{0}(\Omega, K)$. For a given $v \in E$, the support of it is contained in some $\Omega_{k}$, and thus

$$
\begin{aligned}
\left\langle g\left(u_{n}\right), v\right\rangle_{0, \Omega, K} & =\left\langle u_{n}+T\left(u_{n}\right), v\right\rangle_{0, \Omega, K} \\
& =\left\langle u_{n}+T_{m}\left(u_{n}\right), v\right\rangle_{0, \Omega, K} \\
= & \left\langle u_{n}+T_{k}\left(u_{n}\right), v\right\rangle_{0, \Omega, K} \\
& \forall m \geq k, \forall n \in \mathbb{N}
\end{aligned}
$$

by Lemma 11 (c). For each fixed $n \in \mathbb{N}$, there exists $\tilde{n} \in \mathbb{N}$ such that $u_{n} \in C_{0}^{\infty}\left(\Omega_{\widetilde{n}}\right)$. Hence, Lemma $11(\mathrm{e})$ yields

$$
\begin{aligned}
\left\langle g\left(u_{n}\right), v\right\rangle_{0, \Omega, K} & =\left\langle u_{n}+T\left(u_{n}\right), v\right\rangle_{0, \Omega, K} \\
& =\left\langle u_{n}+T_{m}\left(u_{n}\right), v\right\rangle_{0, \Omega, K} \\
& =\left\langle u_{n}+T_{k}\left(u_{n}\right), v\right\rangle_{0, \Omega, K} \\
& \forall m \geq \max \{k, \tilde{n}\} .
\end{aligned}
$$

Taking $n \rightarrow \infty$ in both sides of $\left\langle g\left(u_{n}\right), v\right\rangle_{0, \Omega, K}=\left\langle u_{n}+\right.$ $\left.T_{k}\left(u_{n}\right), v\right\rangle_{0, \Omega, K}$ and using (57) and Lemma 11(d), we deduce

$$
\left\langle u+T_{m}(u), v\right\rangle_{0, \Omega, K}=0, \quad \forall m \geq k .
$$

For any given $v \in C_{0}^{\infty}(\Omega)$, by Lemma 11(b), we have an integer $k$ and a sequence $\left\{v_{l}\right\}_{l} \subset E$ such that $\operatorname{Supp}\left(v_{l}\right) \subset \Omega_{k}$ for any $l \in \mathbb{N}$ and that $v_{l} \rightarrow v$ in $X_{0}(\Omega, K)$. So (60) leads to

$$
\begin{aligned}
\left\langle u+T_{m}(u), v\right\rangle_{0, \Omega, K}=\lim _{l \rightarrow \infty}\left\langle u+T_{m}(u), v_{l}\right\rangle_{0, \Omega, K} & =0 \\
\forall m & \geq k ;
\end{aligned}
$$

that is, $\langle u, v\rangle_{0, \Omega, K}-\int_{\Omega_{m}} f(x, u(x)) v(x) d x=0 \forall m \geq k$. Letting $m \rightarrow \infty$, we get

$$
\langle u, v\rangle_{0, \Omega, K}-\int_{\Omega} f(x, u(x)) v(x) d x=0,
$$

which shows that $u$ is a generalized solution. Note that $u$ might be zero! But $u=0$ is not a solution if $b=f(\cdot, 0)$ takes nonzero values on a nonzero measure set. The proof is completed.

\section{Proofs of Corollaries and Examples}

Proof of Corollary 2. Let $\tilde{f}(x, t)=\lambda G(x)|t|^{\nu-2} t+f(x, t)$. It suffices to check that $\widetilde{f}$ satisfies (15)-(17). Clearly, we can assume $\lambda \neq 0$. Let $\rho=l / r$. Since $1<v<2$, we have $(n-2 s) v>n-2 s$ and thus

$$
l>\frac{2 n}{2 n-(n-2 s) v}>\frac{2 n}{n+2 s}=r .
$$

Then, $\rho>1$ and

$$
\begin{aligned}
1 & <(\nu-1) \rho^{\prime}=(\nu-1) \frac{\rho}{\rho-1}=\frac{l}{l-r}<\frac{n+2 s}{n-2 s} \\
& =2_{s}^{*}-1 .
\end{aligned}
$$

By Young's inequality, we obtain

$$
\begin{aligned}
|\lambda G(x)||t|^{\nu-2} t & =|\lambda G(x)||t|^{\nu-1} \\
& \leq \frac{|\lambda|}{\rho}|G(x)|^{\rho}+\frac{|\lambda|}{\rho^{\prime}}|t|^{(\nu-1) \rho^{\prime}} .
\end{aligned}
$$

Note that $G \in L_{\text {loc }}^{l}(\Omega)$ and $r \rho=l$ imply $|G|^{\rho} \in L_{\text {loc }}^{r}(\Omega)$. Let $\tilde{q}-1=\max \left\{q-1,(\nu-1) \rho^{\prime}\right\}$, which sits in $\left(1,2_{s}^{*}-1\right)$. From these and (15), it follows that

$$
\begin{aligned}
|\tilde{f}(x, t)| \leq & a(x)+\frac{|\lambda|}{\rho}|G(x)|^{\rho}+\frac{|\lambda|}{\rho^{\prime}}|t|^{(\nu-1) \rho^{\prime}} \\
& +\alpha|t|^{q-1} \\
\leq & \left(a(x)+\frac{|\lambda|}{\rho}|G(x)|^{\rho}+C\right)+\tilde{\alpha}|t|^{\widetilde{q}-1}
\end{aligned}
$$

a.e. $x \in \Omega, \forall t \in \mathbb{R}$

for some constants $C>0$ and $\widetilde{\alpha}>0$, where Young's inequality is used again. So $\tilde{f}$ satisfies (15). Moreover, $\tilde{b}(x):=\widetilde{f}(x, 0)=$ $f(x, 0)=b(x)$; that is, $\widetilde{f}$ satisfies (16).

Finally, let us check that (17) holds for $\tilde{f}$. If $\lambda G \leq 0$, then

$$
\begin{aligned}
-\tilde{f}(x, t) t & =-\lambda G(x)|t|^{\nu}-f(x, t) t \\
& \geq-\beta|t|^{p}-c(x) .
\end{aligned}
$$

For another case, observe that

$$
\begin{aligned}
-\tilde{f}(x, t) t & =-\lambda G(x)|t|^{\nu}-f(x, t) t \\
& \geq-\beta|t|^{p}-c(x)-(\lambda G)^{+}(x)|t|^{\nu} .
\end{aligned}
$$

Since $\kappa>2 /(2-\nu)>1$, we may choose a real number $\sigma$ in $(2 /(2-\nu), \kappa)$. Let $\sigma^{\prime}=\sigma /(\sigma-1)$. By Young's inequality, we have

$$
(\lambda G)^{+}(x)|t|^{\nu} \leq \frac{1}{\sigma}\left|(\lambda G)^{+}(x)\right|^{\sigma}+\frac{1}{\sigma^{\prime}}|t|^{\nu \sigma^{\prime}} .
$$


Note that $\left|(\lambda G)^{+}\right|^{\sigma} \in L^{1}(\Omega)$ since $\sigma<\kappa$. Moreover, $1<\nu \sigma^{\prime}$ and

$$
\begin{aligned}
\frac{2}{2-\nu} & <\sigma \Longleftrightarrow \frac{2-\nu}{2}>\frac{1}{\sigma} \Longleftrightarrow \frac{2}{\nu}>\frac{\sigma}{\sigma-1}=\sigma^{\prime} \\
& \Longleftrightarrow \nu \sigma^{\prime}<2 .
\end{aligned}
$$

Let $\widetilde{p}=\max \left\{p, \nu \sigma^{\prime}\right\}$, which belongs to [1,2). Using Young's inequality, we can derive

$$
-\beta|t|^{p}-\frac{1}{\sigma^{\prime}}|t|^{\nu \sigma^{\prime}} \geq-\widetilde{\beta}|t|^{\tilde{p}}-C \quad \forall t \in \mathbb{R}
$$

for some constants $\widetilde{\beta}>0$ and $C>0$. Hence, for a.e. $x \in \Omega$ and all $t \in \mathbb{R}$, we have

$$
\begin{aligned}
-\widetilde{f}(x, t) t & =-\lambda G(x)|t|^{\nu}-f(x, t) t \\
& \geq-\widetilde{\beta}|t|^{\tilde{p}}-\widetilde{c}(x),
\end{aligned}
$$

where $\widetilde{c}(x):=c(x)+(1 / \sigma)\left|(\lambda G)^{+}(x)\right|^{\sigma}+C$ belongs to $L^{1}(\Omega)$ as above. This shows that (17) is true for $\tilde{f}$. The desired conclusion follows from Theorem 1 immediately.

Proof of Corollary 3. Let $\tilde{f}(x, t)=\lambda G(x)|t|^{\nu-2} t+f(x, t)$. Since $\lambda G \leq 0$, we see that $\tilde{f}$ satisfies (17) from the above proof. It remains to prove that $\tilde{f}$ satisfies (15)-(16). Let $\vartheta^{\prime}=\mathscr{\vartheta} /(\vartheta-1)$. Note that

$$
\begin{aligned}
\vartheta & >\frac{n+2 s}{n+2 s-(\nu-1)(n-2 s)} \\
& \Longleftrightarrow \frac{n+2 s-(\nu-1)(n-2 s)}{n+2 s}=1-\frac{(\nu-1)(n-2 s)}{n+2 s} \\
& >\frac{1}{\vartheta} \Longleftrightarrow 1-\frac{1}{\vartheta}=\frac{\vartheta-1}{\vartheta}>\frac{(\nu-1)(n-2 s)}{n+2 s} \\
& \Longleftrightarrow \frac{\vartheta(\nu-1)}{\vartheta-1}=(\nu-1) \vartheta^{\prime}<2_{s}^{*}-1=\frac{n+2 s}{n-2 s} .
\end{aligned}
$$

By Young's inequality, we have

$$
|G(x)||t|^{\nu-1} \leq \frac{|G(x)|^{\vartheta}}{\vartheta}+\frac{|t|^{(\nu-1) \vartheta^{\prime}}}{\vartheta^{\prime}} .
$$

Now, $l \geq r \vartheta$ implies $l / \vartheta \geq r$ and $|G|^{9} \in L_{\text {loc }}^{l / 9}(\Omega)$ because $G \in L_{\mathrm{loc}}^{l}(\Omega)$. We obtain $|G|^{9} \in L_{\mathrm{loc}}^{r}(\Omega)$. As in the proof of Corollary 2, using Young's inequality, we may derive from this and (73)-(74) that $\tilde{f}$ satisfies (15) and (16).

Example 1. Let $n \geq 2$ and $\Omega \subset \mathbb{R}^{n}$ be as above. Consider

$$
\begin{aligned}
\mathfrak{\Omega}_{K} u+G(x) \mathcal{u}+h(u)=0 & \text { in } \Omega, \\
u=0 & \text { in } \mathbb{R}^{n} \backslash \Omega,
\end{aligned}
$$

where $G \leq 0$ belongs to $L_{\text {loc }}^{l}(\Omega)$ with $l \in((n+2 s) / 4 s, n / 2 s)$, $h \in C(\mathbb{R})$ is absolutely continuous, $h(0) \neq 0, \sup _{t \in \mathbb{R}} h(t) t<$ $\infty$, and $\left|h^{\prime}(t)\right| \leq e_{1}+e_{2}|t|^{\rho}$, a.e., $t \in \mathbb{R}, 0 \leq \rho<4 s /(n-2 s)$. Then, (75) has a nontrivial generalized solution.

In fact, taking $v=2$ in Corollary 3 , we should require $\vartheta>(n+2 s) / 4 s$. Since $l \in((n+2 s) / 4 s, n / 2 s)$, there is sufficiently small $\epsilon>0$ such that

$$
\begin{aligned}
1+\epsilon & <\frac{n+2 s}{4 s}+\epsilon<l<\frac{n}{2 s}<\frac{n}{2 s}+r \epsilon \\
& =r \frac{n+2 s}{4 s}+r \epsilon .
\end{aligned}
$$

This means that we can take $\vartheta=(n+2 s) / 4 s+\epsilon$. Moreover, $1 \leq \rho+1<1+4 s /(n-2 s)=2_{s}^{*}$, and

$$
\begin{aligned}
|h(t)-h(0)| & =\left|\int_{0}^{t} h^{\prime}(\tau) d \tau\right| \leq e_{1}|t|+\frac{e_{2}}{\rho+1}|t|^{\rho+1} \\
& \leq \frac{e_{1} \rho}{\rho+1}+\frac{e_{1}+e_{2}}{\rho+1}|t|^{\rho+1} \\
-f(x, t) t & =-G(x) t^{2}-h(t) t \geq-\sup _{t \in \mathbb{R}} h(t) t \\
& >-\infty .
\end{aligned}
$$

Hence, (15)-(17) are satisfied for $f(x, t)=G(x) t+h(t)$.

\section{Proof of Theorem 4}

Consider the product Hilbert space $\mathbf{H}=X_{0}\left(\Omega, K_{1}\right) \times$ $X_{0}\left(\Omega, K_{2}\right)$ equipped with inner product

$$
\begin{aligned}
\langle u, v\rangle_{\mathbf{H}} & =\left\langle\left(u_{1}, u_{2}\right),\left(v_{1}, v_{2}\right)\right\rangle_{\mathbf{H}} \\
& =\left\langle u_{1}, v_{1}\right\rangle_{X_{0}, \Omega, K_{1}}+\left\langle u_{2}, v_{2}\right\rangle_{X_{0}, \Omega, K_{2}}
\end{aligned}
$$

for $u=\left(u_{1}, u_{2}\right), v=\left(v_{1}, v_{2}\right) \in \mathbf{H}$. The induced norm is

$$
\|(u, v)\|_{\mathrm{H}}=\left(\|u\|_{0, \Omega, K_{1}}^{2}+\|v\|_{0, \Omega, K_{2}}^{2}\right)^{1 / 2} .
$$

Let $\Omega=\cup_{k=1}^{\infty} \Omega_{k}$ and $E=\cup_{m} E_{m}$ be as in Section 3. For every integer $m \in \mathbb{N}$ let $\mathbf{E}_{m}=\cup_{k+l \leq m}\left(E_{k} \times E_{l}\right)$ and $\mathbf{E}=\cup_{m} \mathbf{E}_{m}$. Denote by $\mathbf{P}_{m}$ the orthogonal projection from $\mathbf{E}$ onto $\mathbf{E}_{m}$. Corresponding to Lemma 11, we have the following.

Lemma 13. (a) $\mathbf{E}$ is dense in $\mathbf{H}$.

(b) For each $u \in C_{0}^{\infty}(\Omega) \times C_{0}^{\infty}(\Omega)$, there are $k \in \mathbb{N}$ and a sequence $\left\{u_{m}\right\}_{m}$ in $\mathbf{E}$ such that the supports of all $u_{m}$ are contained in $\Omega_{k} \times \Omega_{k}$ and that $u_{m} \rightarrow u$ in $\mathbf{H}$ as $m \rightarrow \infty$.

(c) For every $m \in \mathbb{N}$, and for every given $u \in \mathbf{H}$, there exists a unique $\mathbf{T}_{m}(u)$ in $\mathbf{H}$ such that

$$
\begin{aligned}
\left\langle\mathbf{T}_{m}(u), v\right\rangle_{\mathbf{H}}= & -\int_{\Omega_{m}} f_{1}\left(x, u_{2}(x)\right) v_{1}(x) d x \\
& -\int_{\Omega_{m}} f_{2}\left(x, u_{1}(x)\right) v_{2}(x) d x
\end{aligned}
$$

for any $v=\left(v_{1}, v_{2}\right) \in \mathbf{H}$. Moreover, if $v=\left(v_{1}, v_{2}\right) \in C_{0}^{\infty}\left(\Omega_{k}\right) \times$ $C_{0}^{\infty}\left(\Omega_{k}\right)$, then $\left\langle\mathbf{T}_{m}(u), v\right\rangle_{\mathbf{H}}=\left\langle\mathbf{T}_{k}(u), v\right\rangle_{\mathbf{H}} \forall m \geq k$. 
(d) Suppose that a sequence $\left\{u_{k}\right\}_{k} \subset \mathbf{H}$ weakly converges to $u$ in $\mathbf{H}$. Then, $\left\{\mathbf{T}_{m}\left(u_{k}\right)\right\}_{k}$ weakly converges to $\mathbf{T}_{m}(u)$ in $\mathbf{H}$ for $m=1,2, \ldots$.

(e) For every given $u \in \mathbf{E}$ (the support of $u$ must be contained in some $\Omega_{m_{0}} \times \Omega_{m_{0}}$ by the construction of $\mathbf{E}$ ), there exists a unique $\mathbf{T}(u) \in \mathbf{H}$ such that

$$
\begin{aligned}
\langle\mathbf{T}(u), v\rangle_{\mathbf{H}}= & -\int_{\Omega} f_{1}\left(x, u_{2}(x)\right) v_{1}(x) d x \\
& -\int_{\Omega} f_{2}\left(x, u_{1}(x)\right) v_{2}(x) d x,
\end{aligned}
$$

$\langle\mathbf{T}(u), v\rangle_{\mathbf{H}}=\left\langle\mathbf{T}_{m}(u), v\right\rangle \quad \forall v \in \mathbf{H}(\Omega), \forall m \geq m_{0}$.

(f) $\mathbf{P}_{m} \circ\left(\left.\mathbf{T}\right|_{\mathbf{E}_{m}}\right): \mathbf{E}_{m} \rightarrow \mathbf{E}_{m}$ is continuous for every $m \in \mathbb{N}$.

(g) There exists a constant $C>0$ such that

$$
\begin{aligned}
& \langle u+T(u), u\rangle_{\mathbf{H}} \geq\|u\|_{\mathbf{H}}^{2}[1 \\
& \quad-\left(\left\|c_{1}\right\|_{L^{1}(\Omega)}+\left\|c_{2}\right\|_{L^{1}(\Omega)}\right)\|u\|_{\mathbf{H}}^{-2} \\
& \left.\quad-C\left(\|u\|_{\mathbf{H}}^{p_{1}-2}+\|u\|_{\mathbf{H}}^{\widehat{p}_{1}-2}+\|u\|_{\mathbf{H}}^{p_{2}-2}+\|u\|_{\mathbf{H}}^{\widehat{p}_{2}-2}\right)\right]
\end{aligned}
$$

for all $u \in E \backslash\{0\}$.

Proof. (a) and (b) follow from Lemma 11(a) and (b) immediately.

(c) By (24), we can write $f_{j}(x, t)=b_{j}(x)+$ $f_{0, j}(x, t) \forall(x, t) \in \Omega \times \mathbb{R}$ as in the proof of Lemma 11(c); then, $f_{0, j}(x, 0)=0 \forall x \in \Omega$, and

$$
\begin{aligned}
&\left|f_{0, j}(x, t)\right| \leq a_{j}(x)+b_{j}(x)+\alpha_{j}|t|^{q_{j}-1} \\
& \forall(x, t) \in \Omega \times \mathbb{R}, j=1,2 .
\end{aligned}
$$

Moreover, for every given $m \in \mathbb{N}$ and $j=1$, 2, we have continuous mappings

$$
L^{r\left(q_{j}-1\right)}(\Omega) \ni u \longmapsto \mathfrak{F}_{m, j}(u) \in L^{r}\left(\Omega_{m}\right),
$$

where $\mathfrak{F}_{m, j}(u)(x)=f_{0, j}(x, u(x))$ for $x \in \Omega_{m}, j=1,2$. Recall that $2_{s}^{*}=2_{s}^{*}$. For $u_{1}, v_{1} \in X_{0}\left(\Omega, K_{1}\right), u_{2}, v_{2} \in X_{0}\left(\Omega, K_{2}\right)$, by Lemma 9 (b) and (c), we have

$$
\begin{array}{r}
v_{j} \in L^{2_{s}^{*}}\left(\Omega_{m}\right), \\
u_{j} \in L^{r\left(q_{i}-1\right)}(\Omega), \\
i, j=1,2 .
\end{array}
$$

Thus, $\mathfrak{F}_{m, 1}\left(u_{2}\right) \in L^{r}\left(\Omega_{m}\right), \mathfrak{F}_{m, 2}\left(u_{1}\right) \in L^{r}\left(\Omega_{m}\right)$, and

$$
\begin{aligned}
& \left|-\int_{\Omega_{m}} f_{0,1}\left(x, u_{2}(x)\right) v_{1}(x) d x\right| \\
& =\left|\int_{\Omega_{m}} \mathfrak{F}_{m, 1}\left(u_{2}\right) v_{1}(x) d x\right| \\
& \quad \leq\left\|\mathfrak{\mho}_{m, 1}\left(u_{2}\right)\right\|_{L^{r}\left(\Omega_{m}\right)}\left\|v_{1}\right\|_{L^{2 *}}, \\
& \left|-\int_{\Omega_{m}} f_{0,2}\left(x, u_{1}(x)\right) v_{2}(x) d x\right| \\
& \quad=\left|\int_{\Omega_{m}} \mathfrak{F}_{m, 2}\left(u_{1}\right) v_{2}(x) d x\right| \\
& \quad \leq\left\|\mathfrak{F}_{m, 2}\left(u_{1}\right)\right\|_{L^{r}\left(\Omega_{m}\right)}\left\|v_{2}\right\|_{L_{s}^{2 *}} .
\end{aligned}
$$

Using Lemma 9(c) again, there exist constants $C>0$ such

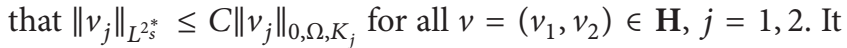
follows that

$$
\begin{aligned}
& \left|-\int_{\Omega_{m}} f_{0,1}\left(x, u_{2}(x)\right) v_{1}(x) d x-\int_{\Omega} b_{1}(x) v_{1}(x) d x\right| \\
& \quad \leq C\left(\left\|\Im_{m, 1}\left(u_{2}\right)\right\|_{L^{r}\left(\Omega_{m}\right)}+\left\|b_{1}\right\|_{L^{r}(\Omega)}\right)\left\|v_{1}\right\|_{0, \Omega, K_{1}}, \\
& \left|-\int_{\Omega_{m}} f_{0,2}\left(x, u_{1}(x)\right) v_{2}(x) d x-\int_{\Omega} b_{2}(x) v_{2}(x) d x\right| \\
& \quad \leq C\left(\left\|\Im_{m, 2}\left(u_{1}\right)\right\|_{L^{r}\left(\Omega_{m}\right)}+\left\|b_{2}\right\|_{L^{r}(\Omega)}\right)\left\|v_{2}\right\|_{0, \Omega, K_{2}} .
\end{aligned}
$$

By the Riesz representative theorem, for each $j \in\{1,2\}$, we have a unique $T_{m j}(u) \in X_{0, \Omega, K_{j}}$ such that

$$
\begin{aligned}
& \left\langle T_{m 1}(u), v_{1}\right\rangle_{0, \Omega, K_{1}}=-\int_{\Omega_{m}} f_{1}\left(x, u_{2}\right) v_{1}(x) d x, \\
& \left\langle T_{m 2}(u), v_{2}\right\rangle_{0, \Omega, K_{2}}=-\int_{\Omega_{m}} f_{2}\left(x, u_{1}\right) v_{2}(x) d x .
\end{aligned}
$$

Setting $\mathbf{T}_{m}(u):=\left(T_{m 1}(u), T_{m 2}(u)\right)$, we obtain

$$
\begin{aligned}
\left\langle\mathbf{T}_{m}(u), v\right\rangle_{\mathbf{H}}= & \left\langle T_{m 1}(u), v_{1}\right\rangle_{0, \Omega, K_{1}} \\
& +\left\langle T_{m 2}(u), v_{2}\right\rangle_{0, \Omega, K_{2}} \\
= & -\int_{\Omega_{m}} f_{1}\left(x, u_{2}\right) v_{1}(x) d x \\
& -\int_{\Omega_{m}} f_{2}\left(x, u_{1}\right) v_{2}(x) d x .
\end{aligned}
$$

Another claim can be proved as that of Lemma 11(c).

(d) Let $u_{k}=\left(u_{k}^{1}, u_{k}^{2}\right)$ for each $k \in \mathbb{N}$. Then, $\left\{u_{k}^{1}\right\}_{k} \subset$ $X_{0, \Omega, K_{1}}$ weakly converges to $u^{1}$ in $X_{0}\left(\Omega, K_{1}\right)$ and $\left\{u_{k}^{2}\right\}_{k} \subset$ $X_{0, \Omega, K_{2}}$ weakly converges to $u^{2}$ in $X_{0}\left(\Omega, K_{2}\right)$. For each $m \in$ $\mathbb{N}$, by Lemma $11(\mathrm{~d}),\left\{T_{m 1}\left(u_{k}\right)\right\}_{k}$ weakly converges to $T_{m 1}(u)$ in $X_{0}\left(\Omega, K_{1}\right)$ and $\left\{T_{m 2}\left(u_{k}\right)\right\}_{k}$ weakly converges to $T_{m 2}(u)$ in 
$X_{0}\left(\Omega, K_{2}\right)$. Thus, $\left\{\mathbf{T}_{m}\left(u_{k}\right)\right\}_{k}$ weakly converges to $\mathbf{T}_{m}(u)$ in $\mathbf{H}$ for $m=1,2, \ldots$.

(e) Since $f_{0, j}(x, 0)=0 \forall x \in \Omega, j=1,2$, and $\operatorname{Supp}(u) \subset$ $\Omega_{m_{0}} \times \Omega_{m_{0}}$, for any integer $m \geq m_{0}$ and $v=\left(v_{1}, v_{2}\right) \in \mathbf{H}$, we derive from (89) that

$$
\begin{aligned}
- & \int_{\Omega} f_{1}\left(x, u_{2}(x)\right) v_{1}(x) d x \\
= & -\int_{\Omega} f_{0,1}\left(x, u_{2}(x)\right) v_{1}(x) d x \\
& -\int_{\Omega} b_{1}(x) v_{1}(x) d x \\
= & -\int_{\Omega_{m}} f_{0,1}\left(x, u_{2}(x)\right) v_{1}(x) d x \\
& -\int_{\Omega} b_{1}(x) v_{1}(x) d x=\left\langle T_{m 1}(u), v_{1}\right\rangle_{0, \Omega, K_{1}}, \\
- & f_{2}\left(x, u_{1}(x)\right) v_{2}(x) d x \\
= & -\int_{\Omega} f_{0,2}\left(x, u_{1}(x)\right) v_{2}(x) d x \\
& -\int_{\Omega} b_{2}(x) v_{2}(x) d x \\
= & -\int_{\Omega_{m}} f_{0,2}\left(x, u_{1}(x)\right) v_{2}(x) d x \\
& -b_{2}(x) v_{2}(x) d x=\left\langle T_{m 2}(u), v_{2}\right\rangle_{0, \Omega, K_{2}} .
\end{aligned}
$$

These show that

$$
\begin{aligned}
& X_{0}\left(\Omega, K_{1}\right) \ni v_{1} \longmapsto-\int_{\Omega} f_{1}\left(x, u_{2}(x)\right) v_{1}(x) d x, \\
& X_{0}\left(\Omega, K_{2}\right) \ni v_{2} \longmapsto-\int_{\Omega} f_{2}\left(x, u_{1}(x)\right) v_{2}(x) d x
\end{aligned}
$$

are two continuous linear functionals. Using the Riesz representative theorem again we obtain a unique $T_{1}(u) \epsilon$ $X_{0}\left(\Omega, K_{1}\right), T_{2}(u) \in X_{0}\left(\Omega, K_{2}\right)$ such that

$$
\begin{aligned}
& \left\langle T_{1}(u), v_{1}\right\rangle_{0, \Omega, K_{1}}=-\int_{\Omega} f_{1}\left(x, u_{2}(x)\right) v_{1}(x) d x, \\
& \left\langle T_{2}(u), v_{2}\right\rangle_{0, \Omega, K_{2}}=-\int_{\Omega} f_{2}\left(x, u_{1}(x)\right) v_{2}(x) d x
\end{aligned}
$$

for all $v=\left(v_{1}, v_{2}\right) \in \mathbf{H}$. Set $\mathbf{T}(u):=\left(T_{1}(u), T_{2}(u)\right)$; then,

$$
\begin{aligned}
\langle T(u), v\rangle_{\mathbf{H}}= & -\int_{\Omega} f_{1}\left(x, u_{2}(x)\right) v_{1}(x) d x \\
& -\int_{\Omega} f_{2}\left(x, u_{1}(x)\right) v_{2}(x) d x,
\end{aligned}
$$

$\forall v \in \mathbf{H}$.

Clearly, $\langle\mathbf{T}(u), v\rangle_{\mathbf{H}}=\left\langle\mathbf{T}_{m}(u), v\right\rangle_{\mathbf{H}} \forall v \in \mathbf{H}$ for all $m \geq m_{0}$. (f) follows the above (e) and Lemma 11(f) directly.

(g) Since $1 \leq p_{j}, \widehat{p}_{j}<2<2_{s}^{*}$, by Lemma 9(b) and (c) there is a constant $C_{0}>0$ such that

$$
\begin{aligned}
\left\|u_{i}\right\|_{L^{\hat{p}_{j}}(\Omega)}+\left\|u_{i}\right\|_{L^{p_{j}}(\Omega)} \leq C_{0}\left\|u_{i}\right\|_{0, \Omega, K_{i}} & \\
& \forall u=\left(u_{1}, u_{2}\right) \in \mathbf{H}, i, j=1,2 .
\end{aligned}
$$

It follows from this and (25) that

$$
\begin{aligned}
& \langle u+\mathbf{T}(u), u\rangle_{\mathbf{H}}=\|u\|_{\mathbf{H}}^{2}-\int_{\Omega} f_{1}\left(x, u_{2}(x)\right) u_{1}(x) d x \\
& \quad-\int_{\Omega} f_{2}\left(x, u_{1}(x)\right) u_{2}(x) d x \geq\|u\|_{\mathbf{H}}^{2} \\
& \quad-\int_{\Omega}\left(\beta_{1}\left|u_{2}\right|^{p_{1} / 2}\left|u_{1}\right|^{\widehat{p}_{1} / 2}+\left|c_{1}\right|\right) d x \\
& \quad-\int_{\Omega}\left(\beta_{2}\left|u_{1}\right|^{p_{2} / 2}\left|u_{2}\right|^{\widehat{p}_{2} / 2}+\left|c_{2}\right|\right) d x \geq\|u\|_{\mathbf{H}}^{2} \\
& \quad-\left\|c_{1}\right\|_{L^{1}}-\left\|c_{2}\right\|_{L^{1}}-\frac{\beta_{1}}{2} \int_{\Omega}\left(\left|u_{2}\right|^{p_{1}}+\left|u_{1}\right|^{\widehat{p}_{1}}\right) d x-\frac{\beta_{2}}{2} \\
& \quad \cdot \int_{\Omega}\left(\left|u_{1}\right|^{p_{2}}+\left|u_{2}\right|^{\widehat{p}_{2}}\right) d x \geq\|u\|_{\mathbf{H}}^{2}-\left(\beta_{1}+\beta_{2}\right) \\
& \quad \cdot\left(\left\|u_{1}\right\|_{L^{p_{1}}}^{\widehat{p}_{1}}\right. \\
& \left.+\left\|u_{1}\right\|_{L^{p_{2}}}^{p_{p_{2}}}+\left\|u_{2}\right\|_{L^{p_{1}}}^{p_{1}}+\left\|u_{2}\right\|_{L^{\hat{p}_{2}}}^{\hat{p}_{2}}\right)-\left\|c_{1}\right\|_{L^{1}}-\left\|c_{2}\right\|_{L^{1}} \\
& \quad \geq\|u\|_{\mathbf{H}}^{2}-C\left(\left\|u_{1}\right\|_{0, \Omega, K_{1}}^{\widehat{p}_{1}}+\left\|u_{1}\right\|_{0, \Omega, K_{1}}^{p_{2}}+\left\|u_{2}\right\|_{0, \Omega, K_{2}}^{p_{1}}\right. \\
& \left.+\left\|u_{2}\right\|_{0, \Omega, K_{2}}^{\widehat{p}_{2}}\right)-\left\|c_{1}\right\|_{L^{1}}-\left\|c_{2}\right\|_{L^{1}} \geq\|u\|_{\mathbf{H}}^{2}-C\left(\|u\|_{\mathbf{H}}^{p_{1}}\right. \\
& \left.+\|u\|_{\mathbf{H}}^{\hat{p}_{1}}+\|u\|_{\mathbf{H}}^{p_{2}}+\|u\|_{\mathbf{H}}^{p_{2}}\right)-\left\|c_{1}\right\|_{L^{1}}-\left\|c_{2}\right\|_{L^{1}} \cdot
\end{aligned}
$$

Here, $C=\left(\beta_{1}+\beta_{2}\right) \max \left\{C_{0}^{p_{1}}, C_{0}^{\widehat{p}_{1}}, C_{0}^{p_{2}}, C_{0}^{\widehat{p}_{2}}\right\}$. This leads to $(\mathrm{g})$.

Proof of Theorem 4. We replace the space $X_{0}(\Omega, K)$ in the proof of Theorem 1 by $\mathbf{H}$. Since $p_{j}<2, \widehat{p}_{j}<2$ for $j=1,2$, as in (54), we have $R>0$ such that

$$
\begin{gathered}
1-C\left(R^{p_{1}-2}+R^{\widehat{p}_{1}-2}+R^{p_{2}-2}+R^{\widehat{p}_{2}-2}\right) \\
-\left(\left\|c_{1}\right\|_{L^{1}}+\left\|c_{1}\right\|_{L^{1}}\right) R^{-2}>\frac{1}{4} .
\end{gathered}
$$

Then repeating the proof of Theorem 1 , we get a $u=\left(u_{1}, u_{2}\right) \in$ $\mathbf{H}$ such that $\langle u+\mathbf{T}(u), v\rangle_{\mathbf{H}}=0$ for any $v=(\phi, \psi) \in C_{0}^{\infty}(\Omega) \times$ $C_{0}^{\infty}(\Omega)$; namely, (22) holds.

\section{Conflict of Interests}

The authors declare that there is no conflict of interests regarding the publication of this paper. 


\section{Acknowledgments}

The authors would like to thank the anonymous referee for the valuable comments and suggestions on the paper. This work is partially supported by the NNSF 10971014 and 11271044, of China, and PCSIRT.

\section{References}

[1] X. Ros-Oton, "Nonlocal elliptic equations in bounded domains: a survey," http://arxiv.org/abs/1504.04099.

[2] L. A. Caffarelli and L. Silvestre, "An extension problem related to the fractional Laplacian," Communications in Partial Differential Equations, vol. 32, no. 7-9, pp. 1245-1260, 2007.

[3] B. Barrios, E. Colorado, A. De Pablo, and U. Sanchez, "On some critical problems for the fractional Laplacian operator," Journal of Differential Equations, vol. 252, no. 11, pp. 6133-6162, 2012.

[4] X. Cabré and Y. Sire, "Nonlinear equations for fractional Laplacians, I: regularity, maximum principles, and Hamiltonian estimates," Annales de l'Institut Henri Poincare (C) Non Linear Analysis, vol. 31, no. 1, pp. 23-53, 2014.

[5] X. Cabré and J. Tan, "Positive solutions of nonlinear problems involving the square root of the Laplacian," Advances in Mathematics, vol. 224, no. 5, pp. 2052-2093, 2010.

[6] P. R. Stinga and J. L. Torrea, "Extension problem and Harnack's inequality for some fractional operators," Communications in Partial Differential Equations, vol. 35, no. 11, pp. 2092-2122, 2010.

[7] R. Servadei and E. Valdinoci, "Lewy-Stampacchia type estimates for variational inequalities driven by (non)local operators," Revista Matemática Iberoamericana, vol. 29, no. 3, pp. 1091-1126, 2013.

[8] R. Servadei and E. Valdinoci, "Mountain pass solutions for nonlocal elliptic operators," Journal of Mathematical Analysis and Applications, vol. 389, no. 2, pp. 887-898, 2012.

[9] R. Servadei and E. Valdinoci, "Variational methods for nonlocal operators of elliptic type," Discrete and Continuous Dynamical Systems, Series A, vol. 33, no. 5, pp. 2105-2137, 2013.

[10] R. Servadei and E. Valdinoci, "On the spectrum of two different fractional operators," Proceedings of the Royal Society of Edinburgh Section A: Mathematics, vol. 144, no. 4, pp. 831-855, 2014.

[11] R. Servadei and E. Valdinoci, "The Brezis-Nirenberg result for the fractional Laplacian," Transactions of the American Mathematical Society, vol. 367, no. 1, pp. 67-102, 2015.

[12] A. Fiscella, R. Servadei, and E. Valdinoci, "Density properties for fractional Sobolev spaces," Annales Academiae Scientiarum Fennicae Mathematica, vol. 40, pp. 235-253, 2015.

[13] A. Fiscella, "Saddle point solutions for non-local elliptic operators," Topological Methods in Nonlinear Analysis, vol. 44, no. 2, pp. 527-538, 2014.

[14] A. Fiscella, R. Servadei, and E. Valdinoci, "A resonance problem for non-local elliptic operators," Zeitschrift für Analysis und Ihre Anwendungen, vol. 32, no. 4, pp. 411-431, 2013.

[15] Y. H. Wei and X. F. Su, "Multiplicity of solutions for non-local elliptic equations driven by the fractional Laplacian," Calculus of Variations and Partial Differential Equations, vol. 52, no. 1-2, pp. 95-124, 2015.

[16] P. d'Avenia, M. Squassina, and M. Zenari, "Fractional logarithmic Schrödinger equations," http://arxiv.org/abs/1412.0270.
[17] D. M. Duc, N. H. Loc, and P. V. Tuoc, "Topological degree for a class of operators and applications," Nonlinear Analysis. Theory, Methods \& Applications, vol. 57, no. 4, pp. 505-518, 2004.

[18] E. Di Nezza, G. Palatucci, and E. Valdinoci, "Hitchhiker's guide to the fractional Sobolev spaces," Bulletin des Sciences Mathématiques, vol. 136, no. 5, pp. 521-573, 2012.

[19] P. Drabek and J. Milota, Methods of Nonlinear Analysis. Applications to Differential Equations, Birkhäuser Advanced Texts: Basler Lehrbücher, Birkhäuser, Basel, Switzerland, 2007.

[20] M. A. Krasnosel'kii, Topological Methods in the Theory of Nonlinear Integral Equations, Pergamon Press, Oxford, UK, 1964. 


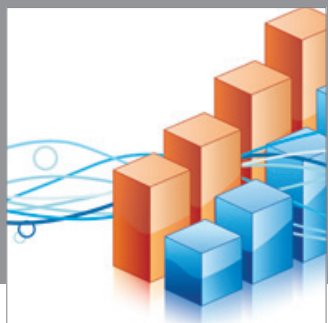

Advances in

Operations Research

mansans

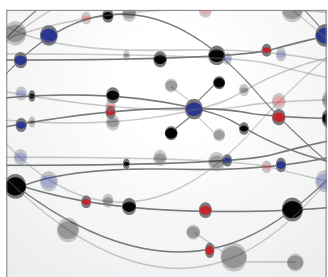

The Scientific World Journal
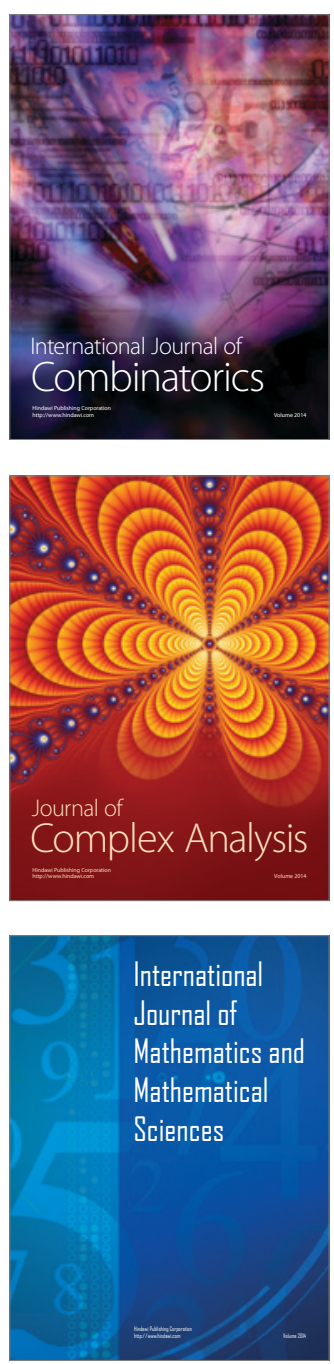
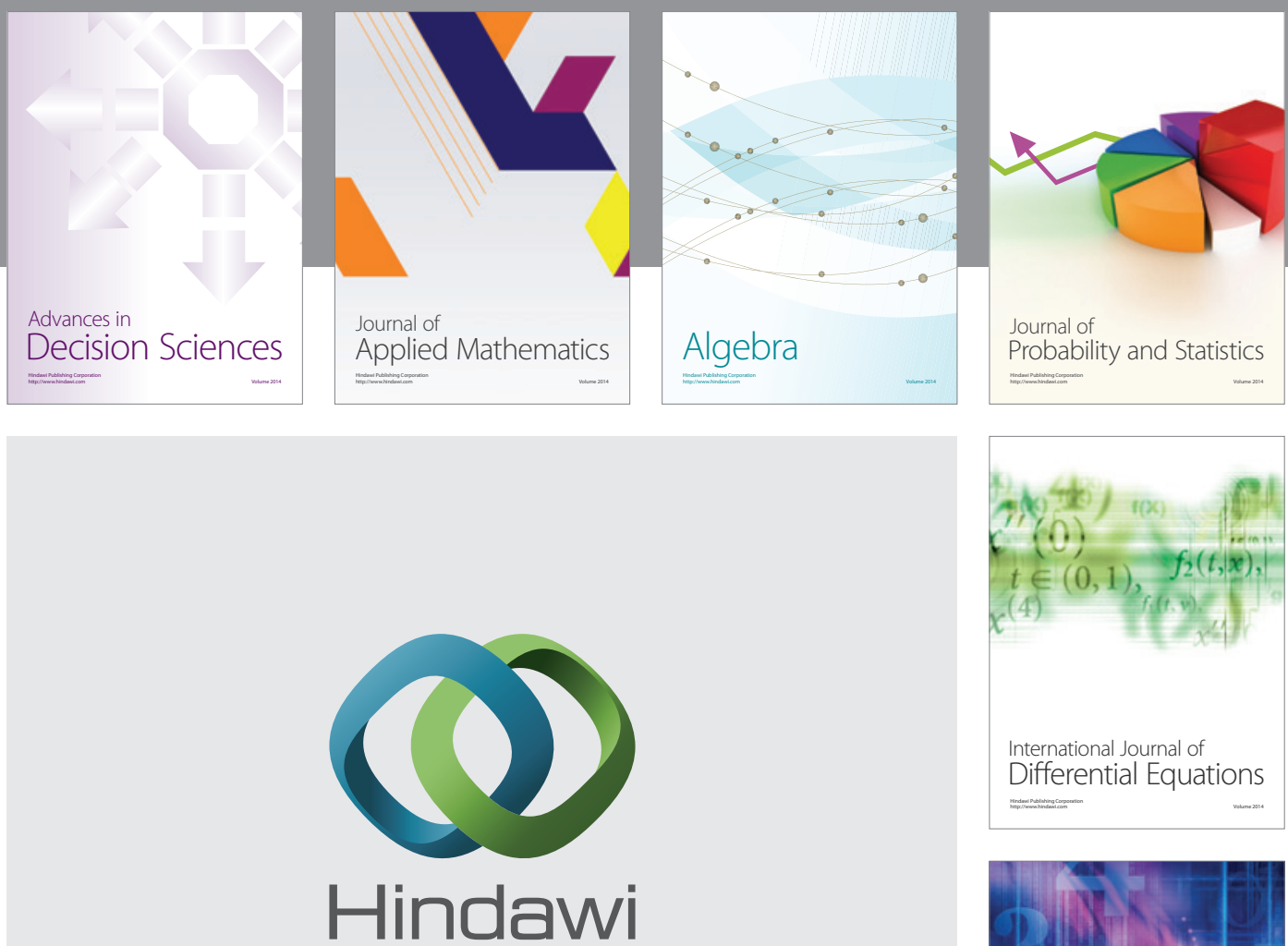

Submit your manuscripts at http://www.hindawi.com
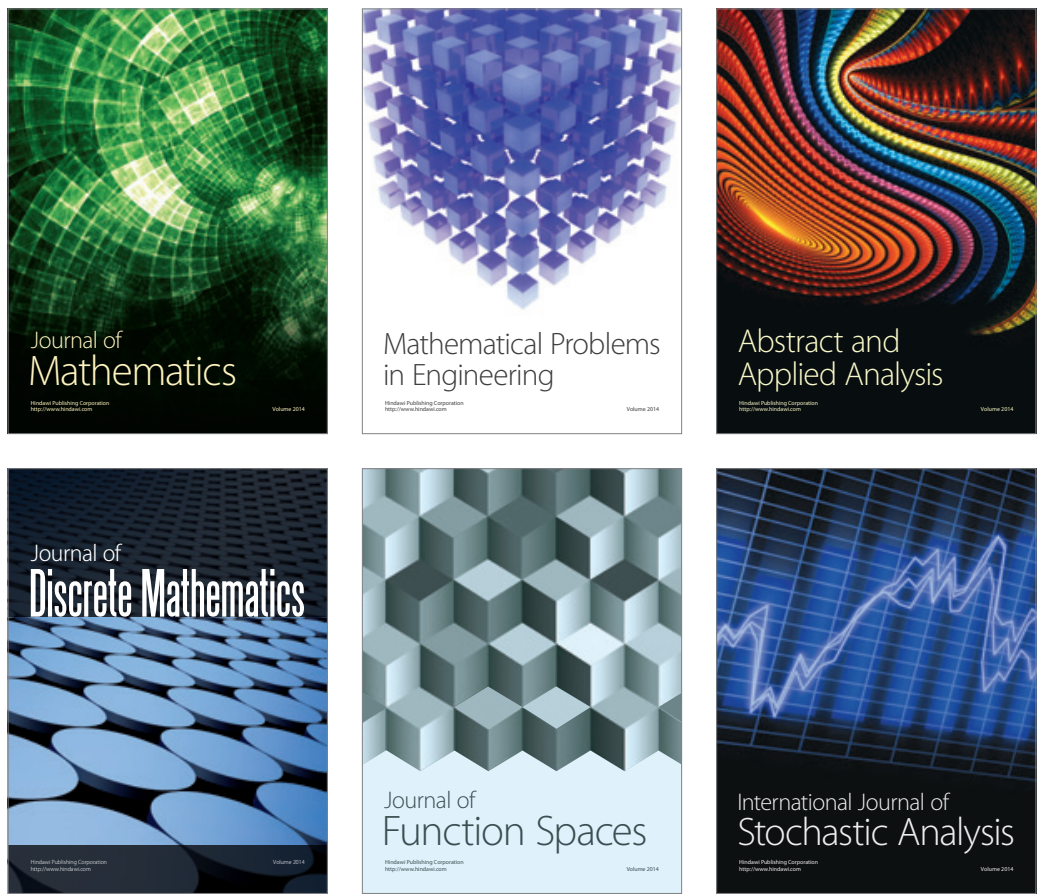

Journal of

Function Spaces

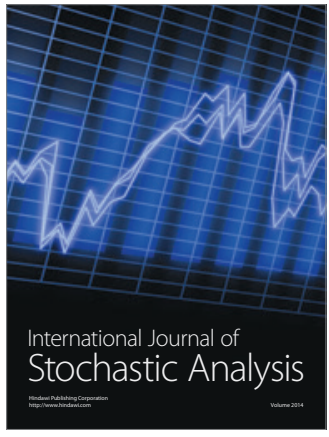

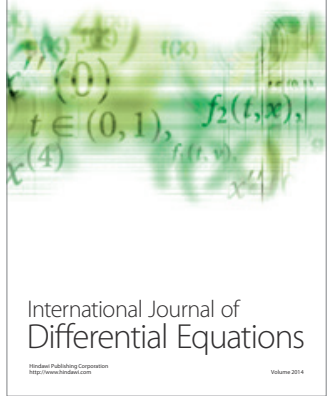
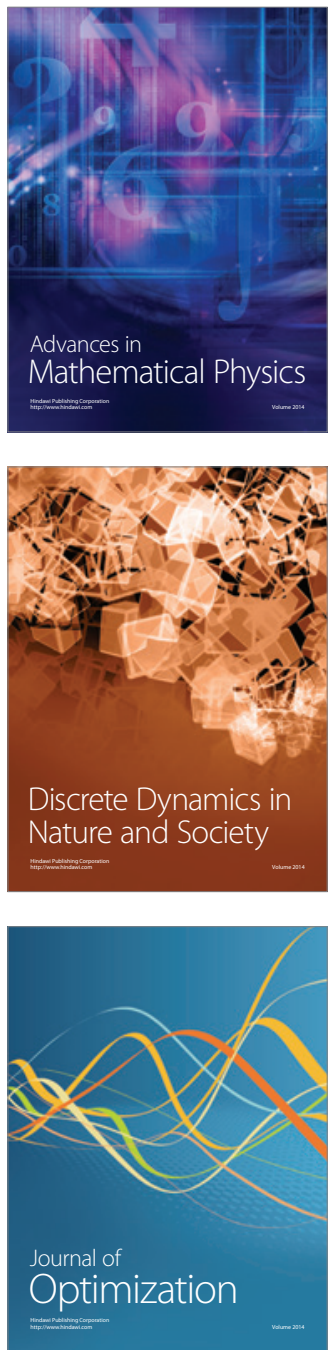